\title{
BMJ Open Healthy Foundations Study: a randomised controlled trial to evaluate biological embedding of early-life experiences
}

\author{
Andrea Gonzalez, ${ }^{1}$ Nicole Catherine, ${ }^{2}$ Michael Boyle, ${ }^{3}$ Susan M Jack, ${ }^{4}$ \\ Leslie Atkinson, ${ }^{5}$ Michael Kobor, ${ }^{6}$ Debbie Sheehan, ${ }^{7}$ Lil Tonmyr, ${ }^{8}$ \\ Charlotte Waddell, ${ }^{9}$ Harriet L MacMillan, ${ }^{10}$ on behalf of the Healthy Foundations \\ Study Team
}

To cite: Gonzalez A, Catherine N, Boyle M, et al. Healthy Foundations Study: a randomised controlled trial to evaluate biological embedding of early-life experiences. BMJ Open 2018;8:e018915. doi:10.1136/ bmjopen-2017-018915

- Prepublication history for this paper is available online. To view these files, please visit the journal online (http://dx.doi. org/10.1136/bmjopen-2017018915).

Received 31 July 2017 Revised 6 December 2017 Accepted 8 December 2017

Check for updates

For numbered affiliations see end of article.

Correspondence to Dr Andrea Gonzalez; gonzal@mcmaster.ca

\section{ABSTRACT}

Introduction Adverse early experiences are associated with long-lasting disruptions in physiology, development and health. These experiences may be 'biologically embedded' into molecular and genomic systems that determine later expressions of vulnerability. Most studies to date have not examined whether preventive interventions can potentially reverse biological embedding. The Nurse-Family Partnership (NFP) is an evidence-based intervention with demonstrated efficacy in improving prenatal health, parenting and child functioning. The Healthy Foundations Study is an innovative birth cohort which will evaluate the impact of the NFP on biological outcomes of mothers and their infants.

Methods and analysis Starting in 2013, up to 400 pregnant mothers and their newborns were recruited from the British Columbia Healthy Connections Project-a randomised controlled trial of the NFP, and will be followed to child aged 2 years. Women were recruited prior to 28 weeks gestation and then individually randomised to receive existing services (comparison group) or NFP plus existing services (intervention group). Hair samples are collected from mothers at baseline and 2 months post partum to measure physiological stress. Saliva samples are collected from infants during all visits for analyses of stress and immune function. Buccal swabs are collected from infants at 2 and 24 months to assess DNA methylation. Biological samples will be related to child outcome measures at age 2 years. Ethics and dissemination The study received ethical approval from seven research ethics boards. Findings from this study will be shared broadly with the research community through peer-reviewed publications, and conference presentations, as well as seminars with our policy partners and relevant healthcare providers. The outcomes of this study will provide all stakeholders with important information regarding how early adversity may lead to health and behavioural disparities and how these may be altered through early interventions.

Trial registration number NCT01672060; Pre-results.

\section{INTRODUCTION}

Adverse early experiences have enduring effects on cognitive, mental and physical health outcomes, ${ }^{1-5}$ with implications for the
Strengths and limitations of this study

- First biological evaluation in infants of preventive intervention using a randomised controlled trial.

- Assesses multiple biomarkers, including DNA methylation and stress physiology.

- Biomarker collection can be challenging as infants get older.

next generation. ${ }^{6}$ Parallel lines of animal and human research show that early adversity produces long-lasting disruptions in physiology, including alterations in the stress system $^{5-7}$ and changes in immune system function, ${ }^{8}{ }^{9}$ which in turn adversely affect brain development and health. ${ }^{7-9}$ These experiences may be 'biologically embedded' into molecular and genomic systems that determine later expressions of vulnerability $^{3-7}$; however, the varied mechanisms and their inter-relations are not fully elucidated. These early experiences set developmental trajectories which canalise over time ${ }^{8}$ and become increasingly difficult to reverse. ${ }^{7}$ Recently, 46 pregnancy and birth cohort studies across Canada were documented by the Maternal Infant Child and Youth Research Network. ${ }^{10}$ While these studies provide important aetiological, descriptive and surveillance data about early risk factors for subsequent negative outcomes, to our knowledge no study has yet examined the impact of preventive interventions, starting in pregnancy and extending postnatally, on biological outcomes in mothers and their children.

To better understand whether a preventive intervention can potentially reverse the biological embedding, we proposed to recruit and follow prospectively a sample of young 
women and their children from within a randomised controlled trial (RCT) evaluating the Nurse-Family Partnership (NFP) programme in a Canadian contextknown as the British Columbia Healthy Connections Project (BCHCP). The NFP is an evidence-based nurse home visitation programme aimed at improving the lives of young, first-time mothers and their children who are experiencing socioeconomic disadvantage. The NFP has demonstrated effectiveness in improving prenatal health, parenting and child development and functioning based on trials conducted in the USA and the Netherlands. ${ }^{11}$ The implementation and delivery of this public health intervention is being evaluated across five unique health authorities (HA) in a mixed-methods process study. ${ }^{12}$ The BCHCP seeks to evaluate the impact of the NFP on health and social outcomes, including childhood injuries (primary outcome), early child development and maternal life course to assess its effectiveness for the first time in Canada. ${ }^{13}$ The substudy of the BCHCP, the Healthy Foundations Study (HFS), is examining the impact of the NFP on biological outcomes in women and their infants followed to age 24 months. A greater understanding of the biology of early adversity and the potential to mitigate detrimental effects will provide a powerful framework with which to inform basic and applied research, practice and policy.

\section{Study rationale}

Risk factors in young mothers and their children

Young, low-income mothers and their children are at high risk of suffering health, educational and economic disparities. In Canada, young mothers $(<24$ years $)$ and their children represent about $5 \%$ of live births each year and constitute one of the most marginalised groups within society. ${ }^{14}$ There is a strong association between young maternal age and socioeconomic deprivation, and higher reported levels of intimate partner violence (IPV) ${ }^{15}$ as well as high-risk behaviours such as smoking, street drug use and poor prenatal care. ${ }^{15-18}$ These maternal risk factors can initiate a trajectory for impaired child development that systematically lowers functional ability during the early years. In brief, children born to these young mothers are at increased risk for cognitive difficulties, impairment in educational and social outcomes, including school adjustment problems, ${ }^{19-23}$ and poorer physical and mental health outcomes. ${ }^{18} 20$ There is an urgent need for effective early interventions for at least two reasons: (1) these trajectories are established early and tend to be reinforced over time; and (2) the ability to change behaviour and brain plasticity decreases over time, likely increasing the costs of implementing successful programmes and lessening the opportunity for realising benefits. ${ }^{24}$

\section{Theoretical and conceptual framework}

To conceptualise the early origins of disparities in learning, behaviour and health, this study is informed by a biodevelopmental framework proposed by Shonkoff. ${ }^{25}$
In this framework, childhood disorders and impairments in learning and behaviour due to early adverse experiences (both prenatal and postnatal) are mediated by alterations in physiological systems, the hypothalamic-pituitary-adrenal (HPA) axis and immune function, and through epigenetic modifications. ${ }^{25}{ }^{26}$ We focus on these factors for two reasons. First, HPA axis function, inflammatory processes and epigenetic changes are three of four most likely mediators of the biological embedding of early experiences (the other is neural structure and function). ${ }^{27}$ Second, animal and human research link these mechanisms to prenatal and postnatal risk factors as well as various offspring outcomes.

The most widely studied mechanism explaining associations between prenatal stress and child outcomes involves the HPA axis. Considerable evidence from both animal and human work demonstrates that exposure to prenatal adversity, including maternal stress, maternal substance use and smoking, exposure to IPV and poverty, and postnatal adversity, such as suboptimal maternal care, are all associated with HPA axis dysregulation. ${ }^{28-40}$ Alterations in HPA axis function are one of the hypothesised mediators between early adversity and later mental and physical health outcomes. ${ }^{41-43}$ Various intervention studies in children and adolescents have shown a positive impact on HPA axis functioning in children exposed to early adverse rearing environments including: foster care children,${ }^{445}$ adopted institutionalised children, ${ }^{46}$ maltreated children, ${ }^{47} 48$ children at risk for antisocial behaviour problems, ${ }^{49}$ parentally bereaved childre ${ }^{50}$ and infants whose mothers were at risk for antenatal depression. ${ }^{51}$ In almost all cases, the impact was reflected in beneficial alterations in the HPA axis (eg, prevention of a blunted cortisol response) in the intervention group compared with the non-intervention group. ${ }^{52}$

Acute psychological stressors, including prenatal stress, exposure to poverty, child maltreatment, bullying, low-quality child care and maternal distress-all result in increases in proinflammatory cytokines, such as C-reactive protein (CRP) and interleukin-6 (IL-6) ${ }^{53-56}$ Inflammatory markers have also been linked to externalising problems ${ }^{57}$ negative mood as well as affective and interpersonal problems in adolescents, ${ }^{58}$ as well as executive dysfunction in obese children. ${ }^{59}$ There is emerging evidence that psychological interventions may have the potential to influence inflammatory markers. For example, in a sample of foster care children enrolled in a Cognitively Based Compassion programme, within the intervention group, practice sessions were associated with decreased CRP levels. ${ }^{60}$ No studies to date have examined whether a preventive intervention, starting prenatally, has an impact on child inflammatory markers and child health.

Recent discoveries in the field of epigenetics have led to a greater understanding of how environmental experiences become biologically embedded. Emerging evidence suggests that early-life experiences may cause epigenetic modifications of the genome (such as changes 
Sources of Early Adversity

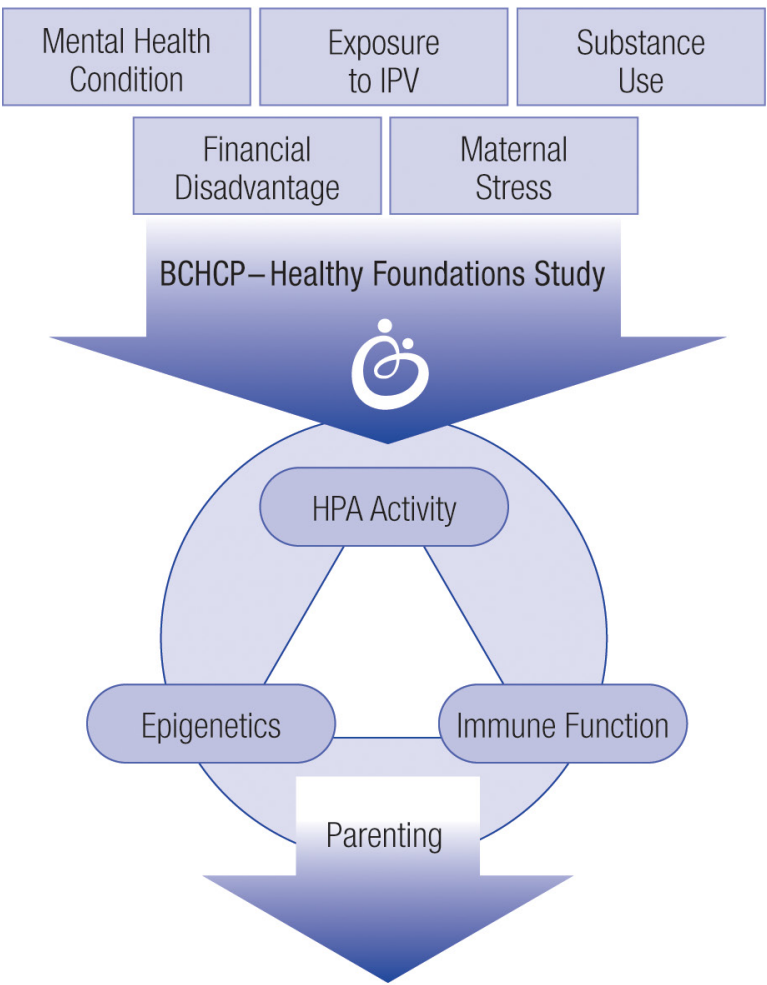

Child Health \& Developmental Outcomes

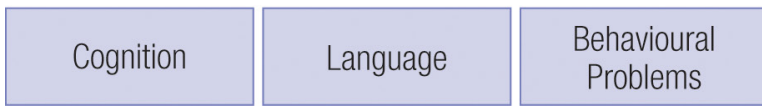

Figure 1 Conceptual model of Healthy Foundations Study (HFS). BCHCP, British Columbia Healthy Connections Project; HPA, hypothalamic-pituitary axis; IPV, intimate partner violence.

in DNA methylation; DNAm), which influence the development of mental and physical health throughout the lifespan. $^{61}{ }^{62}$ Variations in DNAm patterns have been associated with maternal cigarette smoking during pregnancy, ${ }^{6364}$ antenatal depression, ${ }^{65}{ }^{66}$ low socioeconomic status in childhood, ${ }^{6768}$ institutionalisation ${ }^{69}$ and childhood adversity, including maltreatment. ${ }^{70}$ In addition, differential DNAm is associated with variations in cortisol response in infants, ${ }^{71}$ children ${ }^{72}$ and adults, ${ }^{73}$ across a range of child outcomes. ${ }^{74-77}$ To date, one study has illustrated that a family-centred intervention had a protective effect on epigenetic ageing through enhanced parenting. ${ }^{78}$ However, no study to date has examined this within a preventive intervention context starting prenatally.

Collectively, these findings illustrate that prenatal exposure to maternal stress, postnatal adversity and non-optimal caregiving may exert programming influences reflected in the child's capabilities to regulate stress and may impact inflammation, and DNAm. Figure 1 depicts a model of key variables in the HFS. We selected risk factors which are most consistently associated with young motherhood and are targeted by NFP. Our risk factors include: maternal cigarette smoking, maternal stress and presence of mental health condition, exposure to IPV and family financial disadvantage-most of which have been associated with dysregulated HPA function in children, immune function (elevated inflammation levels), alterations in DNAm and various child outcomes. Adopting this model within the context of NFP evaluation provides a unique opportunity to examine associations between these key variables, while assessing the biological impact of this programme.

\section{Nurse-Family Partnership}

The NFP is an evidence-based home visitation programme for vulnerable low-income, young mothers and their children developed in the USA. ${ }^{79}$ In Canada, in a few locations where the NFP is being studied, public health nurses (PHN) trained to deliver the NFP provide intensive education and support to mothers during home visits that start in early pregnancy and continue until the child's second birthday. ${ }^{80}$ In one or more of three US RCTs, the NFP has demonstrated improvements in prenatal health behaviours and birth outcomes, reduction in child mortality rates from preventable causes, as well as improvements in sensitive child care. The NFP has also shown consistent effects in improving child functioning. ${ }^{81-83}$ International trials have been conducted in the Netherlands with positive results and England with null findings. ${ }^{84-86}$ Failure to positively replicate the findings in England has been attributed to selection criteria of participants, based solely on age ( $<20$ years), and the frequency and intensity of services received in the control group as 'usual care'. ${ }^{85}$ In the original US trials, programme effects were strongest in families with concentrated disadvantage, and for children whose mothers had few psychological resources to cope with adversity. ${ }^{85} 86$ None of the completed international trials have included biomarkers to date-thus the HFS provides a unique contribution to the literature on the NFP, and preventive interventions more broadly.

\section{Study objectives}

1. To determine whether the NFP has an effect on infant biological function, as reflected by alterations in HPA axis, immune function and epigenetic markers. Specifically, whether the NFP will attenuate disruptions in HPA axis function (infant salivary cortisol) and inflammatory markers sensitive to early adverse experiences and whether the NFP influences DNAm as a function of intervention group status.

2. To investigate whether the intervention has an impact on prenatal risk factors, including maternal prenatal cortisol levels, and whether these changes are associated with alterations in infants' biological markers. Specifically, to determine if maternal prenatal stress (maternal prenatal cortisol and prenatal adversity) is associated with infant stress physiology (cortisol reactivity and inflammatory markers) and DNAm and whether the NFP moderates these associations. 
3. To examine whether alterations in biological markers explain the impact of the intervention on infant health outcomes. Specifically, we will test a mediation model to examine whether attenuation of postnatal adversity, purported to be induced by the NFP, mediates the association between the NFP on child cortisol levels and health outcomes (cognitive and socioemotional functioning).

Recognising that early adversity does not have the same impact on all children, we consider two additional factors: (1) Child sex: Given that epidemiological studies highlight sex differences in the phenotypic expression of health outcomes ${ }^{87}$ and that prenatal programming may be attributed to these observed sex differences in outcomes, ${ }^{88} 89$ sex differences will be considered in all analyses. (2) Parenting is an important mechanism for regulating offspring physiology and behaviour, ${ }^{90-92}$ and is specifically targeted in NFP (Dyadic Assessment of Naturalistic Caregiver-Child Experiences-a clinical observation tool to guide clinical implementation of parenting content of the NFP),${ }^{93}$ thus we will examine parenting as a potential mediator.

\section{METHODS AND ANALYSIS}

\section{Recruitment, ethics and consent: BCHCP}

The BCHCP is a prospective, large-scale, public health RCT (NCT 01672060), evaluating NFP's effectiveness compared with BC's existing perinatal and early childhood services (see ref ${ }^{13}$ for full details on the larger trial). Referrals to the RCT are received from PHNs across four participating regional HAs. Participant inclusion and exclusion criteria are outlined in box. Eligible and consenting participants are randomised using an unpredictable randomised sequence protocol to receive either existing services (comparison group) or the NFP plus existing services (intervention group). Existing services within British Columbia's universal healthcare system vary by HA; however, many include primary healthcare services from physicians or midwives; specialist physician services (ie, obstetrics and gynaecology); public health programmes including pregnancy screening, prenatal classes and brief home visitation (non-NFP) by nurses or paraprofessionals; and various targeted and universal parenting and early child programmes. Numerous social services including child benefits, employment and income assistance, child protection services, shelter and housing support and food banks are provided by various levels of government (municipal, provincial and federal) and charitable organisations. Given that availability of services varies across districts and whether mothers and their children access these services, data on health and social service utilisation are collected at each time from all participants within BCHCP. ${ }^{13}$ The field interviewers are masked to participants' treatment group allocation. The BCHCP recruited a sample of 739 participants from four HAs (from October 2012 to November 2017).
Box British Columbia Healthy Connections Project (BCHCP) participant inclusion/exclusion criteria

\section{Interviews}

Age 24 years or younger

1. First birth. Women are eligible if a previous pregnancy ended in termination, miscarriage or stillbirth, or if previous parenting involved step-parenting only.

2. Less than 28 weeks' gestation. Women are recruited prior to 28 weeks' gestation to ensure that participants randomised to Nurse-Family Partnership (NFP) receive their first home visit by the end of the 28th week of gestation, according to NFP fidelity requirements.

3. Competent to provide informed consent, including conversational competence in English.

4. Experiencing socioeconomic disadvantage.

- Age 19 or younger.

Ages 20-24: Meets two of three indicators: Lone parent, less than grade 12; or low income which requires one or more of: i. Receiving Medical Services Plan Premium Assistance, disability assistance or other income assistance.

ii. Finding it very difficult to live on total household income with respect to food or rent.

iii. Homeless, defined as living on the streets, living in a place not meant as a long-term dwelling (eg, car or tent), staying in a shelter, or staying somewhere temporarily with no permanent address (eg, 'couch surfing').

Women are ineligible to participate if they meet any exclusion criteria at time of baseline interviews:

1. Planning to have the child adopted.

2. Planning to leave the BCHCP catchment area (designated Local Health Areas) for 3 months or longer during the trial.

Recruitment for HFS started in April 2013 and was completed in April 2017. Based on estimates from an ongoing evaluation in the USA with a similar population, and our feasibility pilot conducted in Hamilton, Ontario, we estimated that on average, $14 \%$ of enlisted participants would be lost to follow-up. To compensate for attrition, the HFS recruited a subsample of 459 women drawn exclusively from two of the four participating HAs: the Fraser Health and the Vancouver Coastal Health. In both HAs, the NFP is delivered by PHNs who have received extensive education in the NFP delivery model. The evaluation follows Consolidated Standards of Reporting Trials guidelines and is registered with the US National Institute of Health clinical trials registry.

\section{Data collection}

$\mathrm{BCHCP}$ questionnaires: assessment of maternal and environmental factors

Data for the BCHCP being collected are conducted at baseline (prior to 28 weeks' gestation and immediately prior to randomisation) and then at 34-36 weeks' gestation (telephone interview), and postnatally at 2, 10, 18 and 24 months. During the interviews, trained Scientific Field Interviewers (SFI) collect sociodemographic data through questionnaires on age, racial/cultural group, family structure and education. ${ }^{94}{ }^{95}$ Socioeconomic 
factors including employment, income and financial supports, relationship status and housing stability are also being collected. Nicotine, alcohol and drug use is being recorded. ${ }^{949697}$ Anxiety and depression in the mother are assessed using the Kessler Psychological Distress Scale. ${ }^{97}$ Exposure to IPV is assessed using the Composite Abuse Scale.$^{98}$ Working within the existing BCHCP interview schedule, the HFS involves collection of biological measures from consenting mother-infant dyads during in-person visits. Hair cortisol samples are collected from women at baseline and at 2 months post partum. Infant salivary samples are being collected at 2, 10, 18 and 24 months and infant buccal swabs are collected at 2 and 24 months. Table 1 includes the full list of measures administered in the BCHCP and HFS.

\section{BCHCP child outcomes at 24 months}

To assess child cognitive development at 24 months the Bayley Test of Infant and Toddler Development (Bayley-III) is being administered. Trained SFI administrators are assessing children using the cognitive and language (expressive and receptive) scales. Standardised scores are derived for each of the cognitive and language domains with a mean of 100 and SD of 15. Standard scores will be used in the mediation model. The Bayley-III has demonstrated adequate validity and reliability. ${ }^{99}$ The ASEBA Child Behavior Checklist (CBCL) for ages 1.5-5 years is administered to assess child socioemotional development. The CBCL consists of 99 items rated by mothers concerning issues, disabilities and descriptions of problems and strengths about the child during the past 6 months. Three primary scales (internalising, externalising, total problems) are also scored. T-scores will be used in the mediation model for Aim 3. Test-retest reliability, inter-rater reliability and predictive validity are high. ${ }^{100}$ Child outcome measures at 24 months will be used in the mediation model for the third objective.

\section{Biological samples: primary outcomes for the HFS Maternal hair cortisol}

Hair cortisol analysis is emerging as a biomarker for chronic stress because systemic cortisol is understood to be incorporated into the hair shaft during hair growth. ${ }^{101}$ To measure maternal physiological stress during pregnancy and early in the post partum, SFIs are collecting hair strands, $3 \mathrm{~mm}$ in diameter ( $\approx 80$ strands), taken from the posterior vertex of the head, as close to the scalp as possible. Strands of hair are cut into a $3 \mathrm{~cm}$ segment to reflect cortisol production in the last 3 months. Information regarding frequency of hair washes and chemical hair processes (bleaching, dying and permanent waves) is collected in response to the current debate regarding the effect of these techniques on hair cortisol levels. ${ }^{102}$

\section{Infant salivary cortisol}

Salivary samples are collected from infants at 2, 10, 18 and 24 months of age. Although there is higher intraindividual variability early in the first year of life, ${ }^{103}$ there is evidence that cortisol samples are positively associated across time and stressors throughout the latter first year (>6 months) extending to the second year of life. ${ }^{104} 105$ During each of these visits, samples are collected at baseline and then at 20, and 40 min poststressor. Poststressor time points are designed to capture both peak response and recovery.

\section{Infant stress procedures}

Given that mild physical stressors (eg, physical examination) are associated with the greatest cortisol reactivity response in infants less than 13 weeks of age (Cohen's d: $0.35-1.69)^{105}$; at 2 months, we administer a mock physical exam as a mild physical stressor and ask the mother to change the infant's diaper and clothing. These procedures have been used successfully in previous studies. ${ }^{106} 107$ To elicit cortisol stress reactivity in infants at 10 months, we use the barrier task, ${ }^{108}$ and at 18 months we use a toy frustration task. ${ }^{109}$ These procedures have been validated in eliciting emotional reactivity and infant cortisol reactivity. ${ }^{103} 104110$ At 24 months, samples are collected around the administration of the Bayley cognitive test, as a novel event/task. Although these challenges are not uniformly stressful across infants, ethical considerations preclude the use of extreme stressors in these age groups. ${ }^{105}$ Nevertheless, these challenges have been repeatedly shown to demonstrate individual differences in cortisol reactivity based on factors such as maternal sensitivity, ${ }^{103} 104111$ attachment security, ${ }^{112}$ maternal antenatal depression, ${ }^{113}$ interparental aggression ${ }^{114}$ and poverty. ${ }^{115}$

\section{Infant inflammatory markers}

CRP and IL-6 will be assayed from baseline, post-20 min and post-40 min salivary samples. Our selection of inflammatory markers was based on a review of the literature and the following criteria: (1) measurable in saliva in children in response to a stressor; (2) associated with prenatal and/or postnatal adversity; and (3) associated with at least one child outcome of interest. Samples will be assayed in duplicate using ELISA with the Salivary ELISA kits from Salimetrics. All markers are detectable in saliva of neonates and older children and can predict abnormal serum thresholds. ${ }^{116} 117$

\section{Behavioural observations of parenting}

The mother and infant are videotaped at 2, 10, 18 and 24 months post partum. Subjects are videotaped interacting during a series of structured interactions. The mother-infant interaction is $4 \mathrm{~min}$ without toys, $4 \mathrm{~min}$ with toys, followed by a $4 \mathrm{~min}$ divided attention task. Mothers are asked to interact as they normally would while remaining within the camera's view. During the last 4 min period, mothers complete a questionnaire while still caring for the infant; the purpose of this task is to place the mother in a divided attention situation thus creating a more ecologically valid situation. The videos will be coded using the Maternal Behaviour Q-Set Revised (MBQS-R $)^{118}$ consisting of 25 items that are sorted and compared with the sort of 
Table 1 Measures and timing of measures in BCHCP and HFS

\begin{tabular}{|c|c|c|c|c|c|c|}
\hline & \multirow[b]{2}{*}{ Construct } & \multicolumn{2}{|c|}{ Prenatal } & \multicolumn{3}{|c|}{ Postnatal } \\
\hline & & Baseline & 2 months & 10 months & 18 months & 24 months \\
\hline & BCHCP measures & & & & & \\
\hline \multirow{21}{*}{$\begin{array}{l}\text { Maternal } \\
\text { measures }\end{array}$} & Sociodemographics & & & & & \\
\hline & Age, racial/cultural group & $x$ & & & & \\
\hline & Prenatal substance use & & & & & \\
\hline & Alcohol use & $x$ & $x$ & $x$ & $x$ & $x$ \\
\hline & Other substance use & $x$ & $x$ & $x$ & $x$ & $x$ \\
\hline & Tobacco use & $x$ & $x$ & $x$ & $\mathrm{x}$ & $x$ \\
\hline & Postnatal substance use & & & & & \\
\hline & Alcohol use & & $x$ & $x$ & $x$ & $x$ \\
\hline & Other substance use & & $x$ & $x$ & $x$ & $\mathrm{x}$ \\
\hline & Tobacco use & & $x$ & $x$ & $x$ & $x$ \\
\hline & Second-hand smoke & & $x$ & $x$ & $x$ & $x$ \\
\hline & Mental health & & & & & \\
\hline & Anxiety and depression & $x$ & $x$ & $x$ & $x$ & $x$ \\
\hline & Financial resources & & & & & \\
\hline & Income and basic needs & $x$ & $x$ & $x$ & $x$ & $x$ \\
\hline & Education, employment & $\mathrm{x}$ & $x$ & $x$ & $x$ & $x$ \\
\hline & Exposure to IPV & & & & & \\
\hline & Intimate partner violence & $x$ & $x$ & $x$ & $x$ & $x$ \\
\hline & Housing & & & & & \\
\hline & $\begin{array}{l}\text { Residential composition and } \\
\text { stability }\end{array}$ & $x$ & $x$ & $x$ & $x$ & $x$ \\
\hline & Secondary outcomes for media & del & & & & \\
\hline \multirow{6}{*}{$\begin{array}{l}\text { Child } \\
\text { measures }\end{array}$} & Child outcome & & & & & \\
\hline & Cognitive development & & & & & $x$ \\
\hline & Child outcome & & & & & \\
\hline & Child behaviour problems & & & & & $x$ \\
\hline & Child outcome & & & & & \\
\hline & Language & & & & & $x$ \\
\hline
\end{tabular}

HFS measures Biomarkers

\begin{tabular}{|c|c|c|c|c|c|c|c|}
\hline \multirow{6}{*}{$\begin{array}{l}\text { Biological } \\
\text { measures }\end{array}$} & \multicolumn{7}{|l|}{ Primary outcomes } \\
\hline & Maternal physiological stress & Maternal hair cortisol & $x$ & $x$ & & & \\
\hline & \multirow[t]{3}{*}{ Infant biological outcomes } & Salivary cortisol levels & & $x$ & $x$ & $x$ & $x$ \\
\hline & & Salivary CRP & & $x$ & $x$ & $x$ & $\mathrm{x}$ \\
\hline & & $\begin{array}{l}\text { DNA methylation } \\
\text { (buccal swabs) }\end{array}$ & & $x$ & & & $x$ \\
\hline & Parenting & Maternal sensitivity & & $x$ & $x$ & $x$ & $x$ \\
\hline
\end{tabular}

BCHCP, British Columbia Healthy Connections Project; CRP, C-reactive protein; HFS, Healthy Foundations Study; IPV, intimate partner violence.

a prototypically sensitive mother. Ultimately, the MBQS-R yields a single score reflecting the mother's responsivity and sensitivity to infant signals. Results from a recent study indicate that the MBQS-R is a reliable and valid measure of the quality of maternal interactive behaviour during a short play session at 10 months post partum. ${ }^{119}$
Epigenetics and DNAm

The plasticity of epigenetic modifications makes it an attractive mechanism at the interface between environment and genome. For example, the best understood epigenetic marker, DNAm, varies with age, gender, tissues and complex disease phenotypes. Importantly, DNAm 
is highly changeable during prenatal development and early life and thus provides an excellent potential biological substrate related to intervention effects. At 2 and 24 months, infant buccal epithelial cells will be collected using Simhelix Dri-Capsules (Boca Scientific). We collect samples at 2 months to capture NFP programme effects prenatally, and then at 24 months to capitalise on the impact of the full intervention on DNAm. Similar to the majority of epigenetic studies in the paediatric age range, we will assess methylation in DNA derived from buccal cells. Compared with blood, buccal cells have the following two important features supporting their use in this study. First, on a practical level, and of particular importance when working with young children, their collection is much less invasive than venipuncture necessary to obtain blood, which increases the likelihood of participation and facilitates retention. Second, on a biological level, they originate from the same embryonic germ layer as the brain. However, despite this common origin, DNAm between buccal cells and brain likely is still highly discordant, given the fundamental role of DNAm in specifying tissue identity during development. Despite the highly tissue-specific nature of DNAm, both in terms of interindividual variability and absolute measures, there are specific CpGs that correlate tightly between peripheral tissues and central tissues. For example, concordant DNAm signatures of ageing have been reported between blood and brain. Perhaps more importantly, an increasing body of research is documenting DNAm signatures related to child development and early-life environments in buccal cells. This then provides a broad comparative basis for DNAm measures performed in the HFS, which will be further expanded by ongoing epigenetic studies in buccal cells in major related cohorts.

\section{Statistical power}

We focus our power analysis on the latent growth curve (LGC) models with mediation. These analyses are most resource intensive, requiring a larger sample size than other objectives to meet power requirements. Calculation of power and sample size for these models is complex because specialised software does not exist. ${ }^{120}$ In addition, as is often the case for such models, ${ }^{121}$ it is difficult to specify the strength of association among the constituent variables. We surveyed articles examining each manifest variable comprising a given latent variable (eg, smoking, maternal mental health, and so on) as they related to the child outcomes of interest here. Ultimately, we decided on a general effect size of $\mathrm{r}=0.30-0.40$, what Cohen $^{122}$ considered a medium-to-large association. This figure seemed to reflect the pertinent literature reasonably well and the selection of a narrow range of effect sizes simplified power analyses considerably. A power study by Fan ${ }^{123}$ demonstrated that a sample size of 200 participants per group is required to detect medium-sized intercept and slope differences between the NFP and control groups using LGC. We then conducted a Monte Carlo simulation to estimate sample size requirements to detect a significant mediational pathway (parallel process) with latent variables using multiple-group structural equation modelling (SEM), $1-\beta=0.80 .{ }^{121}$ The simulation was conducted with 10000 replications using Mplus V.6.11. Given the number of parameters involved in the model, we made a 'best guess' (Thoemmes et al, p 515) ${ }^{120}$ at effect size, assuming it to be medium, $\mathrm{R}^{2}=0.13 .{ }^{121} \mathrm{Simu}-$ lation indicated the need for 200 participants per group (NFP and control). We are confident that a sample size of 400 is sufficient to validate all models proposed here.

\section{Statistical analysis}

Normality of all variables will be examined prior to analyses and appropriate transformations will be applied if required. The BCHCP collects a wide range of maternal risk variables which can be grouped according to two developmental periods (prenatal and postnatal). Given that multiple risk factors at each time point may be inter-related and that our proposed latent variables have not been reported in the literature, prior to the main analyses we will first examine variables using exploratory factor analysis to examine whether any manifest variables have a low factor loading and can be dropped from the analyses. Measures will be combined into latent factors using confirmatory factor analysis. The analyses used to achieve each study objective appear below.

\section{Analysis 1: assessment of biomarkers (primary outcome)}

To evaluate the impact of the NFP on infant biological outcomes (cortisol and inflammatory markers), we will use multilevel growth curve models in MLwiN ${ }^{124}$ to estimate the baseline levels (intercepts) and the trajectories (slopes) of biomarkers over sampling time (baseline, post-20 and post-40) for the four assessment visits (2, 10, 18 and 24 months). Both the model intercept and the slope will be specified as random effects and allowed to covary. Using multilevel growth curve analysis to assess NFP influences on salivary outcomes, we will specify two models: (1) an unconditional model to characterise infant cortisol/inflammatory trajectories (model 1) and (2) a model to compare growth trajectories of NFP infants versus control infants (model 2). Model 2 provides the basis to test whether or not there are statistically significant differences (fixed effects) between infants whose mothers are in the NFP versus standard care. Infant sex will be explored as a moderator (model 3) and parenting will be explored as a mediator (model 4$)$.

\section{Analysis 2: moderation of NFP intervention on prenatal adversity}

To examine whether maternal prenatal cortisol levels and prenatal adversity are predictors of infant cortisol reactivity and inflammatory levels we will use LGC models in Mplus V.8.0. ${ }^{125}$ The model will feature the observed construct of maternal prenatal stress (hair cortisol at baseline) and prenatal adversity (measured at baseline). Prenatal cortisol and prenatal adversity will be simultaneously regressed onto the intercept and slope constructs of infant biomarkers. To examine whether group status 
(NFP vs control) acts as a potential moderator between prenatal stress (cortisol and adversity) and infant biology, we will use a multiple-group approach where models will be estimated simultaneously for NFP infants and control infants. Measurement model parameters will be constrained to equal each other in the two models (eg, error variance and factor loadings), but will be tested for differences in structural parameters (eg, means, variances and predictor pathways). Significant interactions will be tested and probed. ${ }^{126}$ Finally, to explore whether there is a specific time point in which there are NFP versus control group differences in cortisol levels, we will rerun the LGC models with the intercept located at 10, 18 and 24 months.

Analysis 3: mediational analyses of NFP intervention on biomarkers and child outcome

To test our mediation model, in the absence of data to inform the shape of the growth trajectory, we will investigate prior to the main analyses whether the growth trajectories of both the mediator and outcome measures are linear or non-linear using varying design vectors. ${ }^{127-129}$ When examining mediation in an LGC model within an intervention context, mediation is supported when the programme changes the level of the mediator, and changes in the mediator then influence the level of the outcome. Testing mediation in a parallel process LGC model involves three steps. ${ }^{127}$ In the first step, growth trajectories of each process (postnatal adversity and cortisol) will be examined to determine whether the trajectory shape fits the data and to examine whether the slopes are different between intervention and control groups. In the second step, LGC models are combined into a single parallel process model in which the mediation effects are tested by relating the intervention condition, the slope of the mediator and the slope of the outcome. In the third step, mediation effects will be tested using the asymmetric CI test. ${ }^{129}$ Because we anticipate a significant shift in the mediation and outcome only within the NFP group, we will examine whether the specification of the trajectory shape is appropriate for both groups. To accomplish this, factor loadings on latent factors and residual variance of the repeated measure at each assessment time point will be specified as equal across both groups. The covariance between the intercept and the slope will be freely estimated and will be allowed to vary between groups. Finally, to examine the association between child outcomes at age 24 months and infant cortisol, postnatal adversity and group status, we will estimate a serial (multiple mediator) model within a SEM framework using Mplus V.8.0. The multiple mediator chain will be specified (intervention $\rightarrow$ postnatal adversity $\rightarrow$ infant biomarker $\rightarrow$ child outcomes), following the logic of our conceptual model (see figure 1). Asymmetric confidence limits will be calculated to test for mediation effects. To test for sex differences, we will examine which regression parameters are substantially different between sexes. We will use SEM to examine the question of whether any intervention-induced changes in parenting are related to changes in child biological functioning, which is related to child outcomes.

\section{Analysis and interpretation of epigenomic data}

The analysis will focus primarily on interrogating DNAm in the context of $\mathrm{CpG}$ dinucleotides, as it currently represents the most accessible and reliable marker for quantitative epigenetic measurements. DNAm studies take advantage of bisulfite treatment of genomic DNA that selectively deaminates cytosine residues that are not methylated, while leaving methylated cytosines intact. Thus, this approach converts epigenetic information to sequence-based information that will be measured in an unbiased genome-wide assay, using the Illumina Infinium MethylationEPIC BeadChip array. This enables the quantitative interrogation of more than 850000 CpG methylation loci per sample, with a throughput of between 96 and 192 samples per week. The array covers all designable RefSeq genes, with CpG island shores, non-island CpGs, CpG islands outside of coding regions and miRNA promoter regions represented. In R statistical software, these data will undergo stringent quality control and normalisation, removal of all low-quality or cross-hybridising probes and, lastly, batch correction using the COMBAT method. Normalised data will then be used for three complimentary analytical approaches. First, in a hypothesis-driven method, we will a priori derive from the array $\mathrm{CpG}$ sites in candidate genes and test these for association with demographics (child sex) and group status. Candidate genes will be derived from a combination of literature review and computational pathway predictions. These will include genes involved in dopamine and serotonin metabolism, HPA axis and others. Second, to take advantage of the tremendous information content on the Illumina EPIC array, we will perform epigenome-wide association studies (EWAS). This approach provides an opportunity to identify novel genes and pathways associated with our key variables of interest. In practice, we will use bioinformatics methods such as DMRcate, which uses a Gaussian kernel smoothing of DNAm across the genome, to identify broader regions of $\mathrm{CpG}$ loci whose DNAm is associated with NFP status. This analysis will be complemented by CpG site-specific differential methylation analysis using moderated t-statistics with empirical Bayesian variation estimation using the Bioconductor R package 'limma'. In both cases, multiple test correction will be done using the Benjamini-Hochberg method and a false discovery rate (FDR) threshold of $\leq 0.05$ and an arbitrary filter of DNAm difference of $\geq 5 \%$ for each CpG will be applied to increase the likelihood of biological significance. Candidate gene and EWAS will be repeated with maternal prenatal cortisol as a variable, using a similar set of approaches. For all variables, the lists of $\mathrm{CpG}$ resulting from the EWAS approaches will be further examined for enrichment of specific genomic features, including advanced bioinformatics approaches that 
incorporate available data such as CpG density, transcription factor binding sites and chromatin features. Furthermore, resultant gene lists will also be interrogated for association with cellular processes, biological pathways and associated functions, using publicly available and validated tools such as ErmineJ. Collectively, this work will enable the identification of DNAm loci and regions associated with NFP status, and reveal whether epigenetic regulation of broader genomic functions underpins the intervention.

\section{Developmental epigenetic clock}

A novel tool that offers a fundamentally different window into epigenetic biology will complement the powerful candidate gene and EWAS approaches. Specifically, motivated by the success of epigenetic age and its deviation from chronological age as a marker for health, ${ }^{130}$ we have created a developmental epigenetic clock. This clock is specific for buccal epithelial cells, DNAm, and has a much higher accuracy for the paediatric age range compared with existing epigenetic clocks for adults. Using our predictive algorithm, we will compute DNAm developmental age for each infant in NFP versus control groups and determine age deviation from chronological age. This will allow us to assess whether the developmental epigenetic age is accelerated according to NFP status.

\section{ETHICS AND DISSEMINATION}

An independent Data and Safety Monitoring Committee monitors the recruitment, participant safety, protocol compliance and data quality. All data collected (questionnaires, videos and biosamples) are deidentified; labelled only with a unique study code and visit date. Videos are encrypted with 7Zip and stored on password-protected computers within locked facilities. Tissue samples are labelled with unique study codes, and stored in locked cabinets and freezers within locked laboratories. These security provisions are in full compliance with the Tri-Council Policy Statement (TCPS-2, Article 5.3).

Findings from this study will be disseminated in several ways and via multiple platforms, including: publishing in peer-reviewed journals; providing presentations at research conferences and meetings with policy partners and practitioners working with mothers and children; providing regular updates to HFS participants through newsletters and website postings at http://nfp.mcmaster. $\mathrm{ca} /$. All results will be reported as analysis of group data.

\section{DISCUSSION}

Mounting scientific evidence illustrates that a common foundation for health, learning and behaviour is laid early in life. In parallel, advances in biological sciences have begun to elucidate potential mechanisms for the association between early adversity and later physical and mental health outcomes. A greater understanding of the biology of early adversity and the potential to reverse its detrimental effects would provide a powerful framework with which to inform basic and applied research, practice and policy. The biodevelopmental approach provides an alluring model of how the above associations are integrated and may be modified through interventions; however, these hypotheses remain relatively unexplored. Prospective studies examining the biological embedding within an intervention context are virtually non-existent. The HFS would be one of the first to examine the associations espoused by the biodevelopmental model within an RCT for a preventive intervention. Using a methodologically rigorous RCT evaluating an evidence-based intervention provides a unique lens through which processes of biological embedding may potentially be averted. Examining HPA function, inflammatory markers and DNAm in infancy also provides us with information on the importance of intervention effects in the short term, which likely have long-term implications. Recognising that some intervention effects may not be apparent until after intervention completion, and that some of the most pronounced positive effects of the NFP were seen at later ages, ${ }^{131} 132$ our long-term plan is to follow this intervention cohort longitudinally during preschool and school-age years. If the NFP can result in a 'healthier biological phenotype', this may suggest a positive impact of intervention at a biological level, and forecast a greater likelihood of future health. Adopting a biodevelopmental approach will provide practitioners and policymakers with a framework for understanding how early adversity may lead to heath and behavioural disparities and how these may be altered through early interventions. Finally, discoveries on the impact of the NFP on biological outcomes will provide policymakers with far-reaching evidence on the influence of early developmental processes.

\section{Author affiliations}

${ }^{1}$ Psychiatry and Behavioural Neurosciences, McMaster University, Hamilton, Ontario, Canada

${ }^{2}$ Children's Health Policy Centre, Simon Fraser University, Vancouver, British Columbia, Canada

${ }^{3}$ Department of Clinical Epidemiology and Biostatistics, McMaster University, Hamilton, Ontario, Canada

${ }^{4}$ Department of Nursing, McMaster University, Hamilton, Ontario, Canada ${ }^{5}$ Department of Psychology, Ryerson University, Toronto, Ontario, Canada

${ }^{6}$ Department of Medical Genetics, University of British Columbia, Vancouver, British Columbia, Canada

${ }^{7}$ Children's Health Policy Centre, Simon Fraser University, Burnaby, British Columbia, Canada

${ }^{8}$ Family Violence Surveillance, Health Promotion and Chronic Disease Prevention Branch, Public Health Agency of Canada, Ottawa, Ontario, Canada

${ }^{9}$ Psychiatry Department, Simon Fraser University, Burnaby, British Columbia, Canada ${ }^{10}$ Psychiatry and Behavioural Neurosciences, McMaster University, Hamilton, Ontario, Canada

Acknowledgements We are grateful to all participants and their families; the BC NFP nurses and supervisors, and our practice and policy partners in the BC Ministry of Health, the BC Ministry of Children and Family Development, and the Fraser and Vancouver Coastal Health Authorities-all of whom have supported the development and implementation of the HFS. We also thank the BCHCP and HFS field interviewers for all of their work in collecting data. 
Collaborators Members of Healthy Foundations Study Team: Dr Mark Ferro, Dr Pablo Nepomanschy, Dr Ronald Barr, Dr Colleen Varcoe, Dr Lenora Marcellus.

Contributors AG, HLM, NC, MB, CW, LA, MK, SMJ, DS and LT made substantial contributions to the conception and design of the BCHCP and/or the HFS. AG prepared the HFS protocol and drafted all sections of the manuscript. HM, MB, LA and MK contributed to specific sections of the manuscript. All authors read and approved the final manuscript.

Funding The Healthy Foundations Study is supported by Canadian Institutes of Health Research (CIHR; grant number MOP\#130496). The BCHCP is funded by the $\mathrm{BC}$ Ministry of Health with support from the $\mathrm{BC}$ Ministry of Children and Family Development. The participating health authorities in the BCHCP (Fraser Health, Interior Health, Island Health and Vancouver Coastal Health) are funding NFP intervention delivery costs. AG holds a CIHR New Investigator Award. NC is supported by the Djavad Mowafaghian Foundation. HM is supported by the Chedoke Chair in Child Psychiatry. MB, MK and CW are supported by the Canada Research Chairs Program.

Disclaimer The views expressed in this publication are those of the authors and do not necessarily reflect the views of the trial funders nor ClHR.

\section{Competing interests None declared.}

Patient consent Not required.

Ethics approval The protocol for recruitment and collection of BCHCP data, and biological samples for the HFS has been approved by seven research ethics boards of all participating institutions: Simon Fraser University, the University of British Columbia, McMaster University, Ryerson University, the Public Health Agency of Canada, and the two participating health authorities (Fraser Health Authority and Vancouver Coastal Health Research Institute). Informed consent was obtained from expectant women (including consent for the baby/babies that the mother is expecting). Women aged 19 years or younger who are competent to provide informed consent are deemed mature minors by the trial's research ethics boards and may consent to participate without parental consent.

Provenance and peer review Not commissioned; externally peer reviewed.

Open Access This is an Open Access article distributed in accordance with the Creative Commons Attribution Non Commercial (CC BY-NC 4.0) license, which permits others to distribute, remix, adapt, build upon this work non-commercially, and license their derivative works on different terms, provided the original work is properly cited and the use is non-commercial. See: http://creativecommons.org/ licenses/by-nc/4.0/

(C) Article author(s) (or their employer(s) unless otherwise stated in the text of the article) 2018. All rights reserved. No commercial use is permitted unless otherwise expressly granted.

\section{REFERENCES}

1. Shonkoff JP, Garner AS. Committee on Psychosocial Aspects of Child and Family Health, Committee on Early Childhood, Adoption, and Dependent Care, Section on Developmental and Behavioral Pediatrics. The lifelong effects of early childhood adversity and toxic stress. Pediatrics 2012;129:e232-46.

2. Felitti VJ, Anda RF, Nordenberg D, et al. Relationship of childhood abuse and household dysfunction to many of the leading causes of death in adults. The Adverse Childhood Experiences (ACE) Study. Am J Prev Med 1998;14:245-58.

3. Power $\mathrm{C}$, Hertzman $\mathrm{C}$. Social and biological pathways linking early life and adult disease. Br Med Bull 1997;53:210-21.

4. Scott KM, Von Korff M, Angermeyer MC, et al. Association of childhood adversities and early-onset mental disorders with adult-onset chronic physical conditions. Arch Gen Psychiatry 2011;68:838-44.

5. Gunnar MR, Donzella B. Social regulation of the cortisol levels in early human development. Psychoneuroendocrinology 2002;27:199-220.

6. Miller GE, Chen E, Zhou ES. If it goes up, must it come down? Chronic stress and the hypothalamic-pituitary-adrenocortical axis in humans. Psychol Bull 2007;133:25-45.

7. Repetti RL, Taylor SE, Seeman TE. Risky families: family social environments and the mental and physical health of offspring. Psychol Bull 2002;128:330-66.

8. Danese A, McEwen BS. Adverse childhood experiences, allostasis, allostatic load, and age-related disease. Physiol Behav 2012;106:29-39.
9. Repetti RL, Robles TF, Reynolds B. Allostatic processes in the family. Dev Psychopathol 2011;23:921-38.

10. Joly MP, Boivin M, Junker A, et al. An inventory of Canadian pregnancy and birth cohort studies: research in progress. $B M C$ Pregnancy Childbirth 2012;12:117.

11. Shonkoff JP. Leveraging the biology of adversity to address the roots of disparities in health and development. Proc Natl Acad Sci U S A 2012;109:17302-7.

12. Jack SM, Sheehan D, Gonzalez A, et al. British Columbia Healthy Connections Project process evaluation: a mixed methods protoco to describe the implementation and delivery of the Nurse-Family Partnership in Canada. BMC Nurs 2015;14:47.

13. Catherine N, Gonzalez A, Boyle M, et al. for the British Columbia Healthy Connections Project. Evaluating Nurse-Family Partnership in Canada: The British Columbia Healthy Connections Project randomized controlled trial protocol. BMC Health Serv Res 2016;16:349.

14. Chen XK, Wen SW, Fleming N, et al. Teenage pregnancy and adverse birth outcomes: a large population based retrospective cohort study. Int J Epidemiol 2007;36:368-73.

15. Public Health Agency of Canada. What Mothers Say: the Canadian Maternity Experiences Survey. Ottawa, 2009.

16. Terry-Humen E, Manlove J, Moore KA. Playing catch-up: How children born to teen mothers fare. Washington, DC: The national campaign to prevent teen pregnancy, 2005.

17. Horwitz SM, Klerman LV, Kuo HS, et al. School-age mothers: predictors of long-term educational and economic outcomes. Pediatrics 1991;87:862-8.

18. Shaw M, Lawlor DA, Najman JM. Teenage children of teenage mothers: psychological, behavioural and health outcomes from an Australian prospective longitudinal study. Soc Sci Med 2006;62:2526-39.

19. Osofsky JD, Hann DM, Peebles C. Adolescent parenthood: Risks and opportunities for mothers and infants. In: Zeanah C, ed. Handbook of infant mental health. New York: Guilford Press, 1993:106-19.

20. Maughan B, Lindelow M. Secular change in psychosocial risks: the case of teenage motherhood. Psychol Med 1997;27:1129-44.

21. Jutte DP, Roos NP, Brownell MD, et al. The ripples of adolescent motherhood: social, educational, and medical outcomes for children of teen and prior teen mothers. Acad Pediatr 2010;10:293-301.

22. Lowell $\mathrm{H}$, Miller DC. Weight gain during pregnancy: adherence to Health Canada's quidelines. Health Rep 2010;21:31-6.

23. Horodynski MA, Silk K, Hsieh G, et al. Tools for teen moms to reduce infant obesity: a randomized clinical trial. BMC Public Health 2015;15:22

24. Leventhal JM, Horwitz SM, Rude C, et al. Maltreatment of children born to teenage mothers: a comparison between the 1960s and 1980s. J Pediatr 1993;122:314-9.

25. Shonkoff JP. Building a new biodevelopmental framework to guide the future of early childhood policy. Child Dev 2010;81:357-67.

26. Shonkoff JP, Boyce WT, McEwen BS. Neuroscience, Molecular Biology, and the childhood roots of health disparities. JAMA 2009;301:2252-9.

27. Rutter M. Achievements and challenges in the biology of environmental effects. Proc Natl Acad Sci U S A 2012;109:17149-53.

28. McDonald SD, Walker M, Perkins SL, et al. The effect of tobacco exposure on the fetal hypothalamic-pituitary-adrenal axis. BJOG 2006;113:1289-95.

29. Bauer CR, Lambert BL, Bann CM, et al. Long-term impact of maternal substance use during pregnancy and extrauterine environmental adversity: stress hormone levels of preadolescent children. Pediatr Res 2011;70:213-9.

30. Marcus S, Lopez JF, McDonough S, et al. Depressive symptoms during pregnancy: impact on neuroendocrine and neonatal outcomes. Infant Behav Dev 2011;34:26-34.

31. Sandman CA, Davis EP, Buss C, et al. Exposure to prenatal psychobiological stress exerts programming influences on the mother and her fetus. Neuroendocrinology 2012;95:8-21.

32. Pearson J, Tarabulsy GM, Bussières EL. Foetal programming and cortisol secretion in early childhood: A meta-analysis of different programming variables. Infant Behav Dev 2015;40:204-15.

33. Martinez-Torteya C, Bogat GA, Levendosky AA, et al. The influence of prenatal intimate partner violence exposure on hypothalamic-pituitary-adrenal axis reactivity and childhood internalizing and externalizing symptoms. Dev Psychopathol 2016;28:1-18.

34. Sturge-Apple ML, Davies PT, Cicchetti D, et al. Interparental violence, maternal emotional unavailability and children's cortisol functioning in family contexts. Dev Psychol 2012;48:237-49. 
35. Saridjan NS, Huizink AC, Koetsier JA, et al. Do social disadvantage and early family adversity affect the diurnal cortisol rhythm in infants? The Generation R Study. Horm Behav 2010;57:247-54.

36. Douglas JL, Harmer CJ. Early morning cortisol response and emotional processing in adults exposed to postnatal depression in infancy. Eur Psychiatry 2011;26:479-81.

37. Gunnar MR, Hostinar CE. The social buffering of the hypothalamicpituitary-adrenocortical axis in humans: Developmental and experiential determinants. Soc Neurosci 2015;10:478-88.

38. Chen E, Cohen S, Miller GE. How low socioeconomic status affects 2-year hormonal trajectories in children. Psychol Sci 2010;21:31-7.

39. Feldman R, Granat A, Pariente C, et al. Maternal depression and anxiety across the postpartum year and infant social engagement, fear regulation, and stress reactivity. J Am Acad Child Adolesc Psychiatry 2009;48:919-27.

40. Koss KJ, George MR, Davies PT, et al. Patterns of children's adrenocortical reactivity to interparental conflict and associations with child adjustment: a growth mixture modeling approach. Dev Psychol 2013;49:317-26.

41. Lupien SJ, McEwen BS, Gunnar MR, et al. Effects of stress throughout the lifespan on the brain, behaviour and cognition. Nat Rev Neurosci 2009;10:434-45.

42. Juster RP, Bizik G, Picard M, et al. A transdisciplinary perspective of chronic stress in relation to psychopathology throughout life span development. Dev Psychopathol 2011;23:725-76.

43. Taylor SE, Karlamangla AS, Friedman EM, et al. Early environment affects neuroendocrine regulation in adulthood. Soc Cogn Affect Neurosci 2011;6:244-51.

44. Dozier M, Peloso E, Lewis E, et al. Effects of an attachment-based intervention on the cortisol production of infants and toddlers in foster care. Dev Psychopathol 2008;20:845-59.

45. Fisher PA, Van Ryzin MJ, Gunnar MR. Mitigating HPA axis dysregulation associated with placement changes in foster care. Psychoneuroendocrinology 2011;36:531-9.

46. Gunnar MR, Morison SJ, Chisholm K, et al. Salivary cortisol levels in children adopted from Romanian orphanages. Dev Psychopathol 2001;13:611-28.

47. Cicchetti D, Rogosch FA, Toth SL, et al. Normalizing the development of cortisol regulation in maltreated infants through preventive interventions. Dev Psychopathol 2011;23:789-800.

48. Graham AM, Yockelson M, Kim HK, et al. Effects of maltreatment and early intervention on diurnal cortisol slope across the start of school: a pilot study. Child Abuse Negl 2012;36:666-70.

49. Brotman LM, Gouley KK, Huang KY, et al. Effects of a psychosocial family-based preventive intervention on cortisol response to a social challenge in preschoolers at high risk for antisocial behavior. Arch Gen Psychiatry 2007;64:1172-9.

50. Luecken LJ, Hagan MJ, Sandler IN, et al. Cortisol levels sixyears after participation in the Family Bereavement Program. Psychoneuroendocrinology 2010;35:785-9.

51. Urizar GG, Muñoz RF. Impact of a prenatal cognitive-behavioral stress management intervention on salivary cortisol levels in low-income mothers and their infants. Psychoneuroendocrinology 2011;36:1480-94.

52. Slopen N, McLaughlin KA, Shonkoff JP. Interventions to improve cortisol regulation in children: a systematic review. Pediatrics 2014;133:312-26.

53. Copeland WE, Wolke D, Lereya ST, et al. Childhood bullying involvement predicts low-grade systemic inflammation into adulthood. Proc Natl Acad Sci U S A 2014;111:7570-5.

54. Azad MB, Lissitsyn Y, Miller GE, et al. Influence of socioeconomic status trajectories on innate immune responsiveness in children. PLoS One 2012;7:e38669.

55. Slopen N, Loucks EB, Appleton AA, et al. Early origins of inflammation: An examination of prenatal and childhood socia adversity in a prospective cohort study. Psychoneuroendocrinology 2015;51:403-13.

56. Baumeister D, Akhatar R, Ciufolini S, et al. Childhood trauma and adulthood inflammation: a meta-analysis of peripheral C-reactive protein, interleukin-6, and tumor necrosis factor- $\alpha$. MolPsychiatry 2015;21:642-9.

57. Slopen N, Kubzansky LD, Koenen KC. Internalizing and externalizing behaviors predict elevated inflammatory markers in childhood. Psychoneuroendocrinology 2013;38:2854-62.

58. Delany FM, Byrne ML, Whittle S, et al. Depression, immune function, and early adrenarche in children. Psychoneuroendocrinology 2016;63:228-34.

59. Miller AL, Lee HJ, Lumeng JC. Obesity-associated biomarkers and executive function in children. Pediatr Res 2015;77:143-7.

60. Pace TW, Negi LT, Dodson-Lavelle B, et al. Engagement with Cognitively-Based Compassion Training is associated with reduced salivary C-reactive protein from before to after training in foster care program adolescents. Psychoneuroendocrinology 2013;38:294-9.

61. Szyf M. The genome- and system-wide response of DNA methylation to early life adversity and its implication on mental health. Can J Psychiatry 2013;58:697-704.

62. Bick J, Naumova O, Hunter S, et al. Childhood adversity and DNA methylation of genes involved in the hypothalamus-pituitary-adrenal axis and immune system: whole-genome and candidate-gene associations. Dev Psychopathol 2012;24:1417-25.

63. Knopik VS, Maccani MA, Francazio S, et al. The epigenetics of maternal cigarette smoking during pregnancy and effects on child development. Dev Psychopathol 2012;24:1377-90.

64. Joubert BR, Felix JF, Yousefi P, et al. DNA Methylation in Newborns and Maternal Smoking in Pregnancy: Genome-wide Consortium Meta-analysis. Am J Hum Genet 2016;98:680-96.

65. Oberlander TF, Weinberg J, Papsdorf M, et al. Prenatal exposure to maternal depression, neonatal methylation of human glucocorticoid receptor gene (NR3C1) and infant cortisol stress responses. Epigenetics 2008;3:97-106.

66. Cicchetti D, Hetzel S, Rogosch FA, et al. Genome-wide DNA methylation in 1-year-old infants of mothers with major depressive disorder. Dev Psychopathol 2016;28:1413-9.

67. Lam LL, Emberly E, Fraser HB, et al. Factors underlying variable DNA methylation in a human community cohort. Proc Natl Acad Sci U S A 2012;109:17253-60.

68. Borghol N, Suderman M, McArdle W, et al. Associations with early-life socio-economic position in adult DNA methylation. Int $J$ Epidemiol 2012;41:62-74.

69. Naumova OY, Lee M, Koposov R, et al. Differential patterns of whole-genome DNA methylation in institutionalized children and children raised by their biological parents. Dev Psychopathol 2012;24:143-55.

70. Drury SS, Sánchez MM, Gonzalez A. When mothering goes awry: challenges and opportunities for utilizing evidence across rodent, nonhuman primate and human studies to better define the biological consequences of negative early caregiving. Horm Behav 2016;77:182-92.

71. Conradt E, Hawes K, Guerin D, et al. The contributions of maternal sensitivity and maternal depressive symptoms to epigenetic processes and neuroendocrine functioning. Child Dev 2016;87:73-85

72. van der Knaap LJ, Oldehinkel AJ, Verhulst FC, et al. Glucocorticoid receptor gene methylation and HPA-axis regulation in adolescents. The TRAILS study. Psychoneuroendocrinology 2015;58:46-50.

73. Houtepen LC, Vinkers CH, Carrillo-Roa T, et al. Genome-wide DNA methylation levels and altered cortisol stress reactivity following childhood trauma in humans. Nat Commun 2016;7:10967.

74. Dadds MR, Moul C, Hawes DJ, et al. Individual differences in childhood behavior disorders associated with epigenetic modulation of the cortisol receptor gene. Child Dev 2015;86:1311-20.

75. Tyrka AR, Price LH, Marsit C, et al. Childhood adversity and epigenetic modulation of the leukocyte glucocorticoid receptor: preliminary findings in healthy adults. PLoS One 2012;7:e30148.

76. Bromer C, Marsit CJ, Armstrong DA, et al. Genetic and epigenetic variation of the glucocorticoid receptor (NR3C1) in placenta and infant neurobehavior. Dev Psychobiol 2013;55:673-83.

77. Conradt E, Lester BM, Appleton AA, et al. The roles of DNA methylation of NR3C1 and $11 \beta-H S D 2$ and exposure to materna mood disorder in utero on newborn neurobehavior. Epigenetics 2013:8:1321-9

78. Brody GH, Yu T, Chen E, et al. Family-centered prevention ameliorates the longitudinal association between risky family processes and epigenetic aging. J Child Psychol Psychiatry 2016;57:566-74.

79. Macmillan HL, Wathen $\mathrm{CN}$, Barlow J, et al. Interventions to prevent child maltreatment and associated impairment. Lancet 2009;373:250-66.

80. Olds DL. The nurse-family partnership: an evidence-based preventive intervention. Infant Ment Health J 2006;27:5-25.

81. Donelan-McCall N, Eckenrode J, Olds DL. Home visiting for the prevention of child maltreatment: lessons learned during the past 20 years. Pediatr Clin North Am 2009;56:389-403.

82. Olds DL, Kitzman HJ, Cole RE, et al. Enduring effects of prenatal and infancy home visiting by nurses on maternal life course and government spending: follow-up of a randomized trial among children at age 12 years. Arch Pediatr Adolesc Med 2010;164:419-24.

83. Kitzman HJ, Olds DL, Cole RE, et al. Enduring effects of prenatal and infancy home visiting by nurses on children: follow-up of a randomized trial among children at age 12 years. Arch PediatrAdolesc Med 2010;164:412-8. 
84. Robling M, Bekkers MJ, Bell K, et al. Effectiveness of a nurse-led intensive home-visitation programme for first-time teenage mothers (Building Blocks): a pragmatic randomised controlled trial. Lancet 2016;387:146-55.

85. Olds D. Building evidence to improve maternal and child health. Lancet 2016;387:1015-107.

86. Mejdoubi J, van den Heijkant SC, van Leerdam FJ, et al. The effect of VoorZorg, the Dutch nurse-family partnership, on child maltreatment and development: a randomized controlled trial. PLOS One 2015;10:1-14.

87. GBD 2013 Mortality and Causes of Death Collaborators. Global, regional, and national age-sex specific all-cause and causespecific mortality for 240 causes of death, 1990-2013: a systematic analysis for the Global Burden of Disease Study 2013. Lancet 2015;385:117-71.

88. Davis EP, Pfaff D. Sexually dimorphic responses to early adversity: implications for affective problems and autism spectrum disorder. Psychoneuroendocrinology 2014;49:11-25.

89. Braithwaite EC, Kundakovic M, Ramchandani PG, et al. Maternal prenatal depressive symptoms predict infant NR3C1 1F and BDNF IV DNA methylation. Epigenetics 2015;10:408-17.

90. Bergman K, Sarkar P, Glover V, et al. Maternal prenatal cortisol and infant cognitive development: moderation by infant-mother attachment. Biol Psychiatry 2010;67:1026-32.

91. Atkinson L, Gonzalez A, Kashy DL, et al. Maternal sensitivity and infant and mother adrenocortical function across challenges. Psychoneuroendocrinology 2013;38:2943-51.

92. Britto PR, Lye SJ, Proulx K, et al. Nurturing care: promoting early childhood development. Lancet 2017;389:91-102.

93. Olds D, Donelan-McCall N, O'Brien R, et al. Improving the nurse-family partnership in community practice. Pediatrics 2013;132:S110-17.

94. Statistics Canada. Census of Population, 2006. 2010. https:// www12.statcan.gc.ca./census-recensement/2006/index-eng.cfm

95. Statistics Canada. National Longitudinal Survey of Children and Youth (Cycle 8): Book 1 parent, child and youth. 2009. http:// www23.statcan.gc.ca/imdb-bmdi/document/4450_D2_T9_V4-eng. pdf (accessed 17 Feb 2017).

96. ITCY Policy Evaluation Project. International tobacco control four country survey: University of Waterloo, Department of Psychology, 2013. http://www. itcproject.org/surveys (accessed 17 Feb 2017).

97. Kessler RC, Andrews G, Colpe LJ, et al. Short screening scales to monitor population prevalences and trends in non-specific psychological distress. Psychol Med 2002;32:959-76.

98. Hegarty K, Bush R, Sheehan M, et al. The composite abuse scale: further development and assessment of reliability and validity of a multidimensional partner abuse measure in clinical settings. Violence Vict 2005;20:529-47.

99. Bayley N. Bayley Scales of Infant and Toddler Development. 3rd edn. San Antonio, TX: Harcourt Assessment, 2006.

100. Sosna T, Mastergeorge A. Compendium of screening tools for early childhood social-emotional development. Sacramento, CA: California Department of Mental Health, 2005.

101. Stalder T, Steudte-Schmiedgen S, Alexander N, et al. Stress-related and basic determinants of hair cortisol in humans: A meta-analysis. Psychoneuroendocrinology 2017;77:261-74.

102. Tollenaar MS, Jansen J, Beijers R, et al. Cortisol in the first year of life: normative values and intra-individual variability. Early Hum Dev 2010;86:13-16.

103. Goldberg S, Levitan R, Leung E, et al. Cortisol concentrations in 12 to 18-month-old infants: stability over time, location, and stressor. Biol Psychiatry 2003;54:719-26.

104. Atkinson L, Gonzalez A, Kashy DA, et al. Maternal sensitivity and infant and mother adrenocortical function across challenges. Psychoneuroendocrinology 2013;38:2943-51.

105. Jansen J, Beijers R, Riksen-Walraven M, et al. Cortisol reactivity in young infants. Psychoneuroendocrinology 2010;35:329-38.

106. Mörelius E, Hellström-Westas L, Carlén C, et al. Is a nappy change stressful to neonates? Early Hum Dev 2006;82:669-76.

107. de Weerth C, Buitelaar JK. Childbirth complications affect young infants' behavior. Eur Child Adolesc Psychiatry 2007;16:379-88.

108. Ursache A, Blair C, Granger DA, et al. Behavioral reactivity to emotion challenge is associated with cortisol reactivity and regulation at 7,15 , and 24 months of age. Dev Psychobiol 2014;56:474-88.
109. Braungart-Rieker JM, Stifter CA. Infants' responses to frustrating situations: continuity and change in reactivity and regulation. Child Dev 1996;67:1767-79.

110. Blair C, Granger DA, Kivlighan KT, et al. Maternal and child contributions to cortisol response to emotional arousal in young children from low-income, rural communities. Dev Psychol 2008;44:1095-109.

111. Conradt E, Ablow J. Infant physiological response to the still-face paradigm: contributions of maternal sensitivity and infants'early regulatory behavior. Infant Behav Dev 2010;33:251-65.

112. Nachmias M, Gunnar M, Mangelsdorf S, et al. Behavioral inhibition and stress reactivity: the moderating role of attachment security. Child Dev 1996;67:508-22.

113. Kaplan LA, Evans L, Monk C. Effects of mothers' prenatal psychiatric status and postnatal caregiving on infant biobehavioral regulation: can prenatal programming be modified? Early Hum Dev 2008;84:249-56.

114. Towe-Goodman NR, Stifter CA, Mills-Koonce WR, et al. the Family Life Project Key Investigators. Interparental aggression and infant patterns of adrenocortical and behavioral stress responses. Dev Psychobiol 2012;54:685-99.

115. Blair C, Raver CC, Granger D, et al. Allostasis and allostatic load in the context of poverty in early childhood. Dev Psychopathol 2011;23:845-57.

116. lyengar A, Paulus Gerlanc DJ, Maron JL. Front Pediatr. 2014;2:131.

117. Ouellet-Morin I, Danese A, Williams B, et al. Validation of a highsensitivity assay for C-reactive protein in human saliva. Brain Behav Immun 2011;25:640-6.

118. Pederson DR, Moran G. A categorical description of infantmother relationships in the home and its relation to $\mathrm{Q}$-sort measures of infant-mother interaction. In: Vaughn B, Waters E, Posada G, Kondo-Ikemura K, eds. Caregiving, cultural, and cognitive perspectives on secure-base behavior and working models: New growing point of attachment theory and research. Monographs of the Society for Research in Child Dev. 2005;60:111-45.

119. Tarabulsy GM, Provost MA, Bordeleau S, et al. Validation of a short version of the maternal behavior Q-set applied to a brief video record of mother-infant interaction. Infant Behav Dev 2009;32:132-6.

120. Thoemmes F, Mackinnon DP, Reiser MR. Power analysis for complex mediational designs using monte carlo methods. Struct Equ Modeling 2010;17:510-34.

121. Snijders TAB. Power and sample size in multilevel modeling. Encyclopedia of Statistics in Behavioral Science 2005;3:1570-3.

122. Cohen J Statistical power analysis for the behavioral sciences. 2nd edn. New Jersey: Lawrence Erlbaum Associates, 1988.

123. Fan X. Power of latent growth modeling for detecting group differences in linear growth trajectory parameters. Struct Equ Modeling 2003;10:380-400.

124. Rashbash J, Steele F, Browne WJ, et al. A User's Guide to MLwiN. Version 2.26. UK, 2012.

125. Muthen LK, Muthen BO. Mplus User's Guide. 7th edn. Los Angeles, CA: Muthen \& Muthen, 2012

126. Preacher KJ, Curran PJ, Bauer DJ. Computational tools for probing interactions in multiple linear regression, multilevel modeling, and latent curve analysis. J Educ Behav Stat 2006;31:437-48.

127. Mackinnon DP. An introduction to statistical mediation analysis. Mahwah, NJ: Lawrence Erlbaum Associates, 2008.

128. Ram N, Grimm K. Using simple and complex growth models to articulate developmental change: Matching theory to method. Int $\mathrm{J}$ Behav Dev 2007;31:303-16.

129. MacKinnon DP, Fritz MS, Williams J, et al. Distribution of the product confidence limits for the indirect effect: program PRODCLIN. Behav Res Methods 2007;39:384-9.

130. Horvath S, Gurven M, Levine ME, et al. An epigenetic clock analysis of race/ethnicity, sex, and coronary heart disease. Genome Biol 2016;17:171.

131. Olds D, Henderson CR, Cole R, et al. Long-term Effects of Nurse Home Visitation on Children's Criminal and Antisocial Behavior. JAMA 1998;280:1238-44.

132. Olds DL, Robinson J, O'Brien R, et al. Home visiting by paraprofessionals and by nurses: a randomized, controlled trial. Pediatrics 2002;110:486-96. 